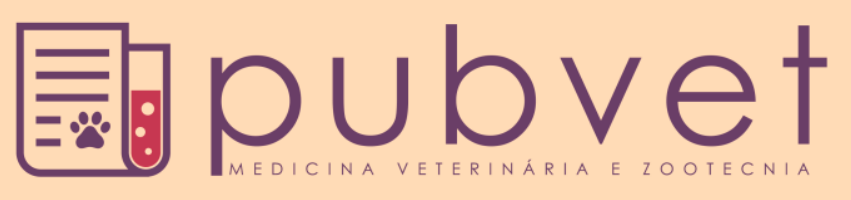

https://doi.org/10.31533/pubvet.v12n11a217.1-7

\title{
Avaliação e caracterização do processo de compostagem de resíduos de peixes
}

\author{
Marcelo dos Santos Nascimento ${ }^{1} \bullet$, Saulo Jander Barbosa Pereira ${ }^{2}$, Renata Franco dos \\ Santos $^{3}{ }^{\circ}$, Auriele Machado Vieira ${ }^{4 *} \bullet$
}

${ }^{1}$ Doutorando do Programa de Pós-Graduando em Aquicultura da Universidade Nilton Lins, Manaus, Amazonas, Brasil ${ }^{2}$ Biólogo pela Universidade Nilton Lins, Manaus, Amazonas, Brasil

${ }^{3}$ Mestranda do Programa de Pós-Graduando em Aquicultura Universidade Nilton Lins, Manaus, Amazonas, Brasil

${ }^{4}$ Mestrando do Programa de Pós-Graduando em Engenharia de Pesca da Universidade Estadual do Oeste do Paraná, Toledo, Paraná, Brasil.

*Autor para correspondência, E-mail: aurieliomv@ hotmail.com

RESUMO. Os resíduos de peixes em sua maior parte são descartes sem nenhum tratamento prévio, desta forma gerando um sério problema ambiental. Um dos meios de tratamento e aproveitamento desses resíduos seria o método da compostagem, que, por meio da biodegradação, tem como função transformar o resíduo em adubo orgânico. Neste contexto, o presente estudo teve como objetivo avaliar e caracterizar o processo de compostagem de resíduos de peixes oriundos de feiras livres, utilizando como fonte de carbono, maravalha, apara de grama e poda de árvores. Os parâmetros avaliados foram: temperatura, umidade, potencial hidrogeniônico $(\mathrm{pH})$, cinzas, matéria orgânica total, carbono orgânico total, nitrogênio total, relação $\mathrm{C} / \mathrm{N}$ e índice de mineralização. Os dados foram submetidos à análise de variância ANOVA e as variáveis que não apresentarão distribuição normal foram analisadas por ANOVA de Kruskall-Wallis e as médias foram comparadas pelo teste de Tukey a 5\% significância. Os resultados dos parâmetros físicosquímicos avaliados ao final do processo de compostagem servem para avaliar a eficácia do processo. Assim, observando que o método de compostagem é uma alternativa viável para o aproveitamento de resíduos de peixe, mesmo os resultados do presente estudo tenham apresentado valores para umidade e relação $\mathrm{C} / \mathrm{N}$ superiores aos recomendados pela legislação brasileira para o uso de fertilizantes orgânicos para ambas os compostos, desta forma, sugerindo o uso de uma maior fonte nitrogênio para a elaboração de compostos orgânicos nas condições experimentais do presente estudo.

Palavras chave: produção, matéria orgânica, biodegradação sustentabilidade

\section{Evaluation and characterization of the fish waste composting process}

ABSTRACT. Most fish waste is discarded without any previous treatment, thus generating a serious environmental problem. One of the means of treatment and recovery of these residues would be the composting method, which, through biodegradation, has the function of transforming the residue into organic fertilizer. In this context, the present study had the objective of evaluating and characterizing the composting process of fish residues from open fairs, using as a source of carbon, shavings, grass cutting and pruning of trees. The parameters evaluated were: temperature, humidity, hydrogen ionic potential $(\mathrm{pH})$, ashes, total organic matter, total organic carbon, total nitrogen, $\mathrm{C} / \mathrm{N}$ ratio and mineralization index. The data were submitted to analysis of variance ANOVA and the variables that will not present normal distribution were analyzed by Kruskall-Wallis ANOVA and the means were compared by the Tukey test at $5 \%$ significance. The results of the physical-chemical parameters evaluated at the end of the composting process are used to evaluate the 
effectiveness of the process. Thus, noting that the composting method is a viable alternative for the use of fish waste, even the results of the present study presented values for humidity and $\mathrm{C} / \mathrm{N}$ ratio higher than those recommended by the Brazilian legislation for the use of organic fertilizers for both the compounds, thus suggesting the use of a larger nitrogen source for the preparation of organic compounds under the experimental conditions of the present study.

Keywords: production, organic matter, biodegradability, sustainability

\section{Evaluación y caracterización del proceso de compostaje de residuos de pescado}

RESUMEN. Los residuos de pescado en su mayor parte son descartes sin ningún tratamiento previo, de esta forma generando un serio problema ambiental. Uno de los medios de tratamiento y aprovechamiento de esos residuos sería el método del compostaje, que, por medio de la biodegradación, tiene como función transformar el residuo en abono orgánico. En este contexto, el presente estudio tuvo como objetivo evaluar y caracterizar el proceso de compostaje de residuos de peces provenientes de ferias libres, utilizando como fuente de carbono, aserrín, recorte de hierba y poda de árboles. Los parámetros evaluados fueron: temperatura, humedad, potencial hidrogenico $(\mathrm{pH})$, cenizas, materia orgánica total, carbono orgánico total, nitrógeno total, relación $\mathrm{C} / \mathrm{N}$ e índice de mineralización. Los datos fueron sometidos al análisis de varianza ANOVA y las variables que no presentarán distribución normal fueron analizadas por ANOVA de Kruskall-Wallis y las medias fueron comparadas por el test de Tukey al 5\% significancia. Los resultados de los parámetros físico-químicos evaluados al final del proceso de compostaje sirven para evaluar la eficacia del proceso. Por lo tanto, observando que el método de compostaje es una alternativa viable para el aprovechamiento de residuos de pescado, incluso los resultados del presente estudio hayan presentado valores para humedad y relación $\mathrm{C} / \mathrm{N}$ superiores a los recomendados por la legislación brasileña para el uso de fertilizantes orgánicos para ambos compuestos, de esta forma, sugiriendo el uso de una mayor fuente de nitrógeno para la elaboración de compuestos orgánicos en las condiciones experimentales del presente estudio.

Palabras clave: producción, materia orgánica, biodegradación, sostenibilidad

\section{Introdução}

A comercialização de alimentos de origem animal em mercados públicos gera uma grande quantidade de lixo orgânico. Em vista, que o crescimento populacional tem agravado o problema do gerenciamento dos resíduos sólidos gerados pelos habitantes, causados, principalmente, pelo volume de lixo produzido e pelo estilo de vida consumista (Oliveira et al. 2012; Bruno et al. 2013).

O termo resíduo refere-se a todos os subprodutos e sobras do processamento de alimentos que são de valor relativamente baixo (Melo et al. 2012). Desta forma, a reciclagem de resíduos, seja de origem agrícola ou industrial, oriundos das mais diversas cadeias produtivas, cujos descartes indevidos podem causar impactos negativos ao ambiente, como é o caso dos resíduos provenientes da indústria pesqueira, apresenta-se como uma importante ferramenta para minimizar o déficit de fertilizantes orgânicos para sistemas produtivos ecológicos (Oliveira et al. 2012; $\underline{\text { Sanes }}$ et al. 2015; Kazemi et al. 2017).

A política de aproveitamento dos resíduos de pescado no Brasil ainda é escassa, sendo que a maioria desses resíduos é descartada, como se observa nas indústrias de beneficiamento de pescado devido, principalmente, à falta de reconhecimento deste recurso como matériaprima e fonte para outros produtos (Pessatti 2001; Nascimento et al. 2014). As cabeças, escamas, peles, vísceras e carcaças (esqueleto com carne aderida) são descartadas durante o processamento e, dependendo da espécie de peixe processada e do produto final obtido pelo frigorífico, estes descartes podem representar algo entre 8 a $16 \%$, (no caso do pescado eviscerado), e 60 a $72 \%$ na produção de filés sem pele (Kubitza \& Campos 2006). Para Oliveira et al. (2006), o descarte desses resíduos de forma incorreta pode causar grandes transtornos ambientais, sanitários e econômicos. Uma alternativa sustentável para o 
aproveitamento dos resíduos de peixes é a compostagem uma vez que pode transformá-los em fertilizante orgânico, servindo como adubo para a plantação de hortaliças por exemplo.

A compostagem é um processo naturalmente controlado, pelo qual os microrganismos benéficos (bactérias e fungos) transformam os resíduos orgânicos em produtos finais estáveis, com baixo risco ambiental e sanitário (Kubitza \& Campos 2006). O processo de compostagem é desenvolvido em duas fases distintas, em que na primeira ocorre a degradação ativa e, na segunda, maturação (humificação) do material orgânico, ocasião em que é produzido o composto propriamente dito (Matos et al. 1998). A compostagem apresenta-se como alternativa viável para sistemas de produção orgânica, em virtude de sua elevada qualidade nutricional e biológica, a elevação dos teores de matéria orgânica, capacidade de troca catiônica, fósforo, potássio, cálcio, magnésio, $\mathrm{pH}$ e saturação por bases permite obter um elevado grau de fertilidade dos solos no sistema orgânico (Sanes et al. 2015). Dessa forma, a compostagem pode ser uma alternativa viável para o aproveitamento dos resíduos de peixes descartados nas feiras e mercados públicos na cidade de Manaus. Neste contexto, o presente estudo teve como objetivo avaliar e caracterizar o processo de compostagem de resíduos de peixes de mercado público, utilizando como fonte de carbono, maravalha, apara de grama e poda de árvores.

\section{Material e Métodos}

O presente estudo foi realizado no setor de Horto da Universidade Nilton Lins (UNL), localizada na cidade de Manaus/AM. O delineamento adotado foi $\mathrm{o}$ inteiramente casualizado com três tratamentos e três repetições. Os tratamentos foram: (CPM) compostagem de resíduo de peixe e maravalha; (CPG) compostagem de resíduo de peixe e aparo de grama e (CPA) compostagem de resíduo de peixe e poda de árvores. O processo de compostagem foi acompanhado durante 40 dias, até a estabilidade da temperatura.

Foram montadas nove composteira de madeira medindo $1,00 \mathrm{~m}$ de comprimento, $0,70 \mathrm{~cm}$ de largura e 0,40 de altura, onde na parte superior as mesmas ficaram abertas e protegidas por lonas plásticas e telhas. As composteiras foram preenchidas de acordo com cada tratamento com as fontes de carbono, maravalha, apara de gramas e podas de árvores e resíduos de peixes na altura de $0,30 \mathrm{~cm}$ respectivamente. As proporções entre as matérias primas foram baseadas em estudos de Liao et al. (1995) e Laos et al. (2002).

A maravalha foi proveniente de madeireiras da região de Manaus, apara de grama e podas de arvores foram coletadas no campus da Universidade Nilton Lins, e os resíduos de peixes (cabeça, carcaça, escamas e vísceras) foram obtidos na feira da Manaus Moderna na cidade de Manaus - AM. A altura utilizada para as camadas do agente de estruturação foi de $0,10 \mathrm{~cm}$, baseado na metodologia de Paiva (2004) determinadas por pesagens e definida por medições com auxílio de uma fita métrica. Resultado no total de $6 \mathrm{~kg}$ de resíduo de peixe para cada composteira.

Durante o processo de compostagem, as mesmas foram reviradas a partir do decimo quinto dia, seguindo novamente a cada cinco dias até o período de estabilização da temperatura das biomassas. A água foi adicionada com o auxílio de um recipiente graduado, na proporção de $30 \%$ da massa de cada biomassa (Costa et al. 2005), o que correspondeu 2,4 L por composteira.

As análises físico-químicas da matéria prima inicial (Tabela 1) e da biomassa final (Tabela. 2), tais como, umidade, nitrogênio total, cinzas e $\mathrm{pH}$, foram realizadas no laboratório de análises de alimentos da Universidade Nilton Lins, determinadas segundo as recomendações da AOAC (2005).

As temperaturas do ambiente (máxima e mínima) para a região foram coletadas no site do Instituto Nacional de Meteorologia (INMET) e a temperatura da biomassa interna e da camada superficial foi realizada diariamente com o auxílio de um termômetro de mercúrio. Umidade das compostagens foi realizada em estufa à uma temperatura de $105^{\circ} \mathrm{C}$, utilizando a equação $\mathrm{U}=$ $100-\%$ de matéria seca. $\mathrm{O} \mathrm{pH}$ foi determinado inicialmente e aos 40 dias, com o auxílio de um pHmetro digital, pela dissolução de $10 \mathrm{~g}$ de amostras, referentes a cada tratamento, em $90 \mathrm{~mL}$ de água a temperatura ambiente. Cinzas foram obtidas por incineração total da matéria orgânica a $600^{\circ} \mathrm{C}$ em forno mufla, por aproximadamente $2 \mathrm{~h}$ será obtido o teor das cinzas até atingir uma cor clara. 
Tabela 1. Composição físico-química dos substratos, resíduo de peixe maravalha, poda de grama e apara de árvores.

\begin{tabular}{lrrrr}
\hline & \multicolumn{3}{c}{ Substrato } \\
\cline { 2 - 5 } Parâmetros & Resíduo de Peixe & Maravalha & Apara de grama & Poda de árvore \\
\hline pH & $6,55 \pm 0,19$ & $6,11 \pm 0,06$ & $6,38 \pm 0,17$ & $6,07 \pm 0,05$ \\
\hline Umidade (\%) & $80,35 \pm 1,58$ & $20,30 \pm 4,10$ & $16,73 \pm 0,10$ & $16,66 \pm 0,11$ \\
Cinzas (\%) & $29,21 \pm 1,39$ & $1,06 \pm 0,03$ & $0,96 \pm 0,01$ & $0,97 \pm 0,33$ \\
Matéria orgânica (\% & $70,78 \pm 1,39$ & $98,93 \pm 0,03$ & $99,04 \pm 0,01$ & $99,14 \pm 0,19$ \\
Carbono orgânico total (\%) & $39,32 \pm 0,77$ & $54,96 \pm 0,02$ & $55,02 \pm 0,00$ & $55,08 \pm 0,01$ \\
Nitrogênio total (\%) & $11,01 \pm 0,03$ & $0,23 \pm 0,21$ & $1,70 \pm 0,08$ & $1,48 \pm 0,02$ \\
Carbono/nitrogênio & $3,57 \pm 0,08$ & $234,41 \pm 21,32$ & $32,26 \pm 0,79$ & $37,18 \pm 2,09$ \\
Índice de mineralização & $0,74 \pm 0,05$ & $0,02 \pm 0,01$ & $0,02 \pm 0,00$ & $0,02 \pm 0,01$
\end{tabular}

Médias submetidos a análise de variância (ANOVA) e teste de Tukey $(\mathrm{P}<0,05)$ de significância. Valores a esquerda da média referem-se aos respectivos desvios-padrões (SD).

A porcentagem do material orgânico presente nas compostagens foi calculada pela diferença do peso entre a matéria seca e as cinzas, conforme a metodologia descrita por Kiehl (2004), sendo que para obter o teor de matéria orgânica foi aplicada a equação $\mathrm{MO}=100-\%$ de cinzas. $\mathrm{O}$ carbono orgânico total foi realizada através da divisão da matéria orgânica total por 1,8 (fator de Bemmelen), aplicando a equação $\mathrm{C}=\mathrm{MO} / 1,80$, seguindo os métodos descritos por Kiehl (2004). Nitrogênio total foi determinado pela digestão da amostra (com ácido sulfúrico), destilação e titulação com o auxílio do aparelho Micro Kjedahl. Relação C/N foi obtida pela equação C/N $=\% \mathrm{C} \div \% \mathrm{~N}$, "onde $\% \mathrm{C}=$ porcentagem de carbono orgânico total na amostra; $\% \mathrm{~N}=$ porcentagem de nitrogênio total na amostra", conforme descrito por Tedesco et al. (1995) e o índice de mineração do composto foi realizado com auxílio da equação $\mathrm{IMC}=\% \mathrm{CZ} \div \% \mathrm{C}$, onde $\% \mathrm{CZ}=\mathrm{A}$ porcentagem de cinzas na amostra; \%C $=\mathrm{A}$ porcentagem de carbono orgânico total da amostra, conforme Drozd et al. (1997).
Para avaliação dos dados resultantes foi aplicado análise de variância (ANOVA) e o teste de Turkey a um nível de significância de 5\%. As variáveis que não apresentarão distribuição normal foram analisadas por ANOVA de Kruskall-Wallis.

\section{Resultados e discussão}

Os valores referentes às temperaturas médias das compostagens com seus respectivos desvios padrões e os valores médios da temperatura ambiente, durante o período de 40 dias estão descritos na figura 1 . Onde podemos observar que a estabilização das biomassas dos compostos de resíduo de peixe e maravalha (CPM), resíduo de peixe e apara de grama (CPG) ocorreu próxima ao trigésimo segundo dia, e no composto de resíduo de peixe e poda de árvore (CPA) ocorreu no trigésimo quinto dia, isso em decorrência a temperatura das biomassas atingirem temperaturas próximas a ambiente, conforme ilustra a Figura 1.

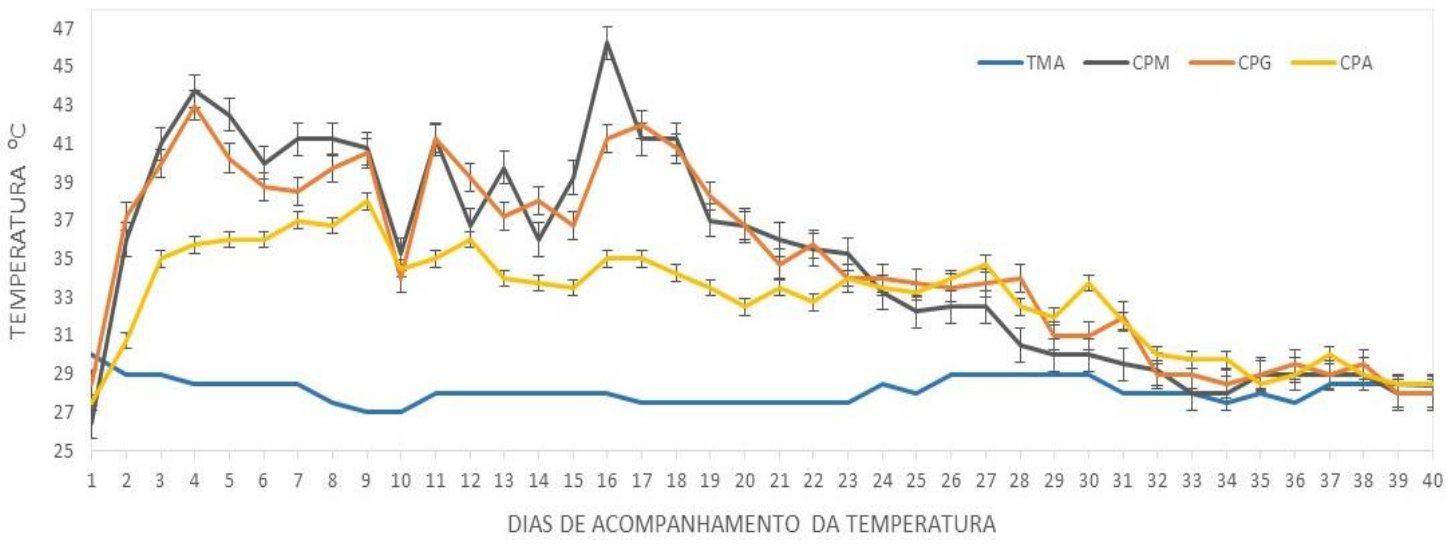

Figura 1. Médias das temperaturas durante o processo de compostagem de residuo de peixe com diferentes fontes de carbono e temperatura média do meio ambiente. TMA: Temperatura média ambiente; CPM: compostagem de residuo de peixe e maravalha. CPG: compostagem de residuo de peixe e apara de grama; CPA: compostagem de residuo de peixe e poda de arvores. 
Observa-se também que as biomassas CPM e CPG nos primeiros dias do processo de compostagem atingiram temperaturas acima de $40^{\circ} \mathrm{C}$, o mesmo não foi observado para a biomassa CPA. Sendo que a biomassa do composto CPM atingiu temperaturas mais altas $\left(46,3^{\circ} \mathrm{C}\right)$ entre o décimo sexto e o décimo sétimo dia. As reduções e variações das temperaturas nas biomassas durante o processo de compostagem podem ter ocorrido devido a presença de chuvas intensas durante 18 dias na região, assim favorecendo a perda de calor e resultando na alta variação de temperatura, em decorrência disso predominando a população de bactérias mesófila durante a maior parte do período experimental em ambos os tratamentos.

O comportamento da temperatura da biomassa observado nos tratamentos é característico dos processos de compostagem, apresentando o mesmo comportamento encontrado por Valente et al. (2016), em compostagem de resíduos de filetagem de pescado e casca de arroz, por Kazemi et al. (2017) sobre compostagem baseada em resíduos de peixes marinhos, como observado por Sanes et al. (2015), em compostagem com resíduo de peixe e casca de acácia.

Segundo Kiehl (2004), logo após a montagem da leira a temperatura pode ser menor do que a do ambiente, devido ao resfriamento provocado pela evaporação da água presente na decomposição da massa, característica essa observada no presente estudo com ambas as biomassas apresentando temperaturas variando entre 26,5 a $27,5^{\circ} \mathrm{C}$, enquanto a temperatura ambiente apresentava-se na faixa de $30^{\circ} \mathrm{C}$. Segundo o mesmo autor, nos dias subsequentes, a decomposição do composto começa a gerar calor e a temperatura começa a subir: nessa ascensão tem-se inicialmente a fase mesófila, seguida de outra mais quente denominada termófila. Prosseguindo a decomposição, a temperatura vai baixar e o composto entrar na fase mesófila novamente.

Os resultados referentes à composição físicoquímica das compostagens estão presentes na tabela 2. Onde podemos observar que não ocorreu diferença significativa $(\mathrm{P}>0,05)$ para os valores de $\mathrm{pH}$, Cinzas, matéria orgânica total e índice de mineralização entre os tratamentos.

Os valores encontrados nos parâmetros avaliados na composição físico-química das compostagens estão próximos aos valores encontrados por Valente et al. (2016), em seu estudo com compostagens de resíduos de filetagens de pescados e casca de arroz. $\mathrm{O} \mathrm{pH}$ e a relação carbono/nitrogênio também apresentaram valores próximos aos encontrados por Kazemi et al. (2017), em sua pesquisa com compostagem baseada em resíduos de pescados marinhos.

Segundo o MAPA - Instrução Normativa $n^{\circ}$ 25 , de 23/07/2009, os valores de $\mathrm{pH}$ mínimo aceitável para a comercialização de composto orgânicos devem ser iguais ou maior que 6,0. Deste modo, para o parâmetro $\mathrm{pH}$, os compostos avaliados neste estudo atendem tal legislação vigente. De acordo Iglesias-Jiménez and Pérez García (1989), valores baixos de pH são indicativos de falta de maturação devido à curta duração do processo ou à ocorrência de processos anaeróbios no interior da pilha em compostagem. Já Kiehl (2004) descreve que o pH fornece informações sobre o estado de decomposição da matéria orgânica, onde a matéria-prima crua tem reação ácida; quando neutra ou quase neutra, o composto está estabilizado, assim, o composto humificado apresentará obrigatoriamente reação alcalina.

Tabela 2. Composição físico-química das compostagens após 40 dias de mistura dos resíduos de peixe e substratos

\begin{tabular}{lccc}
\hline Parâmetros & \multicolumn{3}{c}{ Compostagem } \\
\cline { 2 - 4 } & CPM & CPG & CPA \\
\hline $\mathrm{pH}$ & $7,92 \pm 0,09$ & $8,12 \pm 0,0$ & $8,09 \pm 0,04$ \\
Umidade (\%) & $55,59 \pm 0,59 \mathrm{a}$ & $69,97 \pm 3,18 \mathrm{~b}$ & $69,63 \pm 6,20 \mathrm{~b}$ \\
Cinzas (\%) & $2,58 \pm 0,48$ & $3,28 \pm 1,75$ & $4,72 \pm 0,04$ \\
Matéria orgânica (\%) & $97,42 \pm 0,48$ & $96,72 \pm 1,74$ & $95,28 \pm 0,04$ \\
Carbono orgânico total (\%) & $54,12 \pm 0,26$ & $53,74 \pm 0,97$ & $52,93 \pm 0,02$ \\
Nitrogênio total (\%) & $0,96 \pm 0,45 \mathrm{~b}$ & $1,42 \pm 0,10 \mathrm{a}$ & $1,16 \pm 0,23 \mathrm{ab}$ \\
Relação carbono/nitrogênio & $56,54 \pm 2,41 \mathrm{a}$ & $47,49 \pm 2,72 \mathrm{ab}$ & $37,35 \pm 2,71 \mathrm{~b}$ \\
Índice de mineralização & $0,05 \pm 0,03$ & $0,06 \pm 0,00$ & $0,09 \pm 0,01$ \\
\hline
\end{tabular}

CPM: compostagem de residuo de peixe e maravalha. CPG: compostagem residuo de peixe e apara de grama; CPA: compostagem de residuo de peixe e poda de arvores. Valores a esquerda da média referem-se aos respectivos desvios-padrões (SD). Letras distintas indicam diferenças estatísticas entre as variáveis $(\mathrm{P}<0,05)$. 
De acordo com Drozd et al. (1997), os valores de IMC devem ser maiores que 1,30, pois quando mais alto este valor, maior a mineralização da matéria orgânica, sendo este valor maior dos que os observados para os compostos avaliados (Tabela 2), os resultados do presente estudo corroboram com os obtidos por Valente et al. (2014) que observaram valores de 0,1 para compostagem de resíduo de peixe e maravalha.

No entanto, os valores de umidade para os compostos estão acima dos recomendados pela legislação $(\leq 50 \%)$. Esses valores acima dos recomendados podem ter sidos influenciados pela presença de fortes chuvas que deixavam ao redor das compoteiras inundadas durante 18 dias. Segundo Liang et al. (2003), a umidade é um fator primordial para a atividade metabólica e fisiológica dos microrganismos. Mas valores maiores acima de $50 \%$ podem fazer com que a água ocupe os espaços vazios do meio, impedindo a livre passagem do oxigênio na biomassa do composto.

Para os valores de nitrogênio e relação $\mathrm{C} / \mathrm{N}$ foi observado diferença $(\mathrm{P}>0,05)$ entre os tratamentos. Sendo as medias para nitrogênio total, estando dentro dos valores recomendados pela legislação. Já os valores de relação $\mathrm{C} / \mathrm{N}$ estão acima dos recomendados para o uso de fertilizantes, de acordo com a instrução normativa $n^{0} 25 / 2009$ que sugere uma relação igual ou menor que 20/1. Segundo Kiehl (2004), quando um fertilizante orgânico com uma relação $\mathrm{C} / \mathrm{N}$ alta é aplicado em um cultivo de plantas, os microorganismos retiram $\mathrm{N}$ do solo na forma de $\mathrm{NH} 3$, que estará indisponível para as raízes das plantas.

Os resultados do presente estudo referentes a $\mathrm{C} / \mathrm{N}$ corroboram Zhu (2007) afirma que valores mais elevados de relação $\mathrm{C} / \mathrm{N}$ significam que não há nitrogênio suficiente para um ótimo crescimento das populações microbianas, havendo imobilização de nitrogênio pelos microorganismos, prejudicando assim a velocidade de decomposição dos resíduos orgânicos.

As diferenças significativas $(\mathrm{P}>0,05)$ observadas entre os compostos avaliados sugerem que a composição dos materiais que constituíram as misturas tem influenciado no processo de compostagem (tabela 1). Segundo Kiehl (2004), o acompanhamento da relação $\mathrm{C}: \mathrm{N}$ durante a compostagem permite conhecer o andamento do processo, pois quando o composto atinge a semicura, a relação C:N se situa em torno de 18/1, e quando atinge a maturidade, ou seja transformou-se em produto acabado ou humificado, a relação $\mathrm{C} / \mathrm{N}$ se situa em torno de $10 / 1$.

\section{Conclusão}

Os parâmetros físico-químicos avaliados ao final do processo de compostagem servem para avaliar a eficácia do processo. Assim, observando que o método de compostagem é uma alternativa viável para o aproveitamento de resíduos de peixe, mesmo os resultados do presente estudo tenham apresentado valores para umidade e relação $\mathrm{C} / \mathrm{N}$ superiores aos recomendados pela instrução normativa $\mathrm{n}^{\mathrm{o}}$ 25/2009 do Ministério da Agricultura Pecuária e Abastecimento para o uso de fertilizantes orgânicos para ambas os compostos avaliados, desta forma, sugerindo um substrato com maior fonte nitrogênio para a elaboração de compostos orgânicos nas condições experimentais do presente estudo.

\section{Referências}

AOAC 2005 - Association Official Analytical Chemist (2005). AOAC, Gaitherburg, Maryland, USA.

Bruno F.H.S., Sales R.O., Oliveira A.L.T. \& Freitas J.B.S. 2013) Avaliação de diferentes concentrações de adubo orgânico produzido a partir de resíduos de pescados e vegetais no desenvolvimento da cultura da cebolinha (Allium schoenoprasum). Revista Brasileira de Higiene e Sanidade Animal 7, 86-105.

Costa M.S.S.M., Costa L.A.M., Olibone D., Röder C., Burin A., Kaufmann A.V. \& Ortolan M.L. 2005. Efeito da aeração no primeiro estágio da compostagem de carcaça de aves. Engenharia Agrícola 25, 549-556.

Drozd J., Jamroz E., Licznar M., Licznar S.E. \& Weber J. 1997. Organic matter transformation and humic indices of compost maturity stage during composting of municipal solid wastes. Grunwaldzka 53, 855-861.

Iglesias-Jiménez E. \& Pérez García V. 1989. Evaluation of city refuse compost maturity: a review. Biological Wastes 27, 115-142.

Kazemi K., Zhang B., Lye L.M. \& Zhu Z. 2017. Evaluation of state and evolution of marine fish waste composting by enzyme activities. Canadian Journal of Civil Engineering 44, 348-357.

Kiehl E.J. 2004. Manual de compostagem: maturação e qualidade do composto. Degaspari, Piracicaba. 
Kubitza F. \& Campos J.L. 2006. O aproveitamento dos subprodutos do processamento de pescado. Panorama da aquicultura 16, 23-29.

Laos F., Mazzarino M.J., Walter I., Roselli L., Satti P. \& Moyano S. 2002. Composting of fish offal and biosolids in northwestern Patagonia. Bioresource Technology 81, 179-186.

Liang C., Das K.C. \& McClendon R.W. 2003. The influence of temperature and moisture contents regimes on the aerobic microbial activity of a biosolids composting blend. Bioresource Technology 86, 131-137.

Liao P.H., May A.C. \& Chieng S.T. 1995. Monitoring process efficiency of a full-scale invessel system for composting fisheries wastes. Bioresource Technology 54, 159-163.

Matos A.T., Vidigal S.M., Sediyama M.A.N., Garcia N.C.P. \& Ribeiro M.F. 1998. Compostagem de alguns resíduos orgânicos, utilizando-se águas residuárias da suinocultura como fonte de nitrogênio. Revista Brasileira de Engenharia Agricola e Ambiental 2, 199-203.

Melo F.O., Alves M.M., Guimarães M.D.F. \& Holand F.C.A.F. 2012. Aproveitamento do resíduo a partir do beneficiamento de pescado de uma indústria pesqueira no norte do Brasil. Arquivos de Ciências do Mar 44, 5-11.

Nascimento S.M., Freitas K.F.S. \& Silva M.V. 2014. Produção e caracterização de silagens de resíduos de peixes comercializados no mercado público de Parnaíba-PI. Enciclopédia Biosfera 10, 2458-2466.

Oliveira A.L.T., Oliveira Sales R., Freitas J.B.S. \& Lopes J.E. 2012. Alternativa sustentável para descarte de resíduos de pescado em Fortaleza. Revista Brasileira de Higiene e Sanidade Animal 6, 1-16.

Oliveira M.M., Pimenta M.E.S.G., Camargo A.C.S., Fiorini J.E. \& Pimenta C.J. 2006. Silagem de resíduos da filetagem de tilápia do nilo (Oreochromis niloticus), com ácido fórmico-análise bromatológica, físico-química e microbiológica. Ciência e Agrotecnologia 30, 1218-1223.

Paiva D.P. 2004. Uso da compostagem como destino de suínos mortos e restos de parição. In: Manual de boas práticas (ed. by Oliveira PA), pp. 100-104. EMBRAPA, Concórdia, Santa Catarina, Brasil.

Pessatti M.L. 2001. Aproveitamento dos subprodutos do pescado. Meta 11.

Sanes F.S.M., Strassburger A.S., Araújo F.B. \& Medeiros C.A.B. 2015. Compostagem e fermentação de resíduos de pescado para produção de fertilizantes orgânicos Waste composting and proving fish for production the organic fertilizers. Semina: Ciências Agrárias 36, 1241-1252.

Tedesco M.J., Gianello C., Bissani C.A., Bohnen H. \& Volkweiss S.J. 1995. Análises de solo, plantas e outros materiais. Universidade Federal do Rio Grande do Sul, Porto Alegre, Rio Grande do Sul, Brasil.

Valente B.S., Xavier E.G., Pereira H.d.S. \& Tabeleão Pilotto M.V. 2016. Compostagem de resíduos da filetagem de pescado marinho e casca de arroz. Revista Brasileira de Saúde e Produção Animal 17, 237-248.

Valente B.S., Xavier E.G., Pereira H.S. \& Pilotto M.V.T. 2014. Compostagem na gestão de resíduos de pescado de água doce. Boletim Instituto de Pesca 40, 95-103.

Zhu N. 2007. Effect of low initial $\mathrm{C} / \mathrm{N}$ ratio on aerobic composting of swine manure with rice straw. Bioresource Technology 98, 9-13.

Recebido: 3 Outubro, 2018.

Aprovado: 30 Outubro, 2018.

Publicado: 8 Dezembro, 2018.

Licenciamento: Este artigo é publicado na modalidade Acesso Aberto sob a licença Creative Commons Atribuição 4.0 (CC-BY 4.0), a qual permite uso irrestrito, distribuição, reprodução em qualquer meio, desde que o autor e a fonte sejam devidamente creditados. 